Supporting Information for

\title{
Liquid-Liquid Phase Separation of Peptide/Oligonucleotide Complexes in Crowded Macromolecular Media
}

Qingwen Bai, Qiufen Zhang, Hairong Jing, Jiaxin Chen and Dehai Liang

Beijing National Laboratory for Molecular Sciences, Department of Polymer Science and Engineering and the Key Laboratory of Polymer Chemistry and Physics of the Ministry of Education, College of Chemistry and Molecular Engineering, Peking University, Beijing 100871,

China 


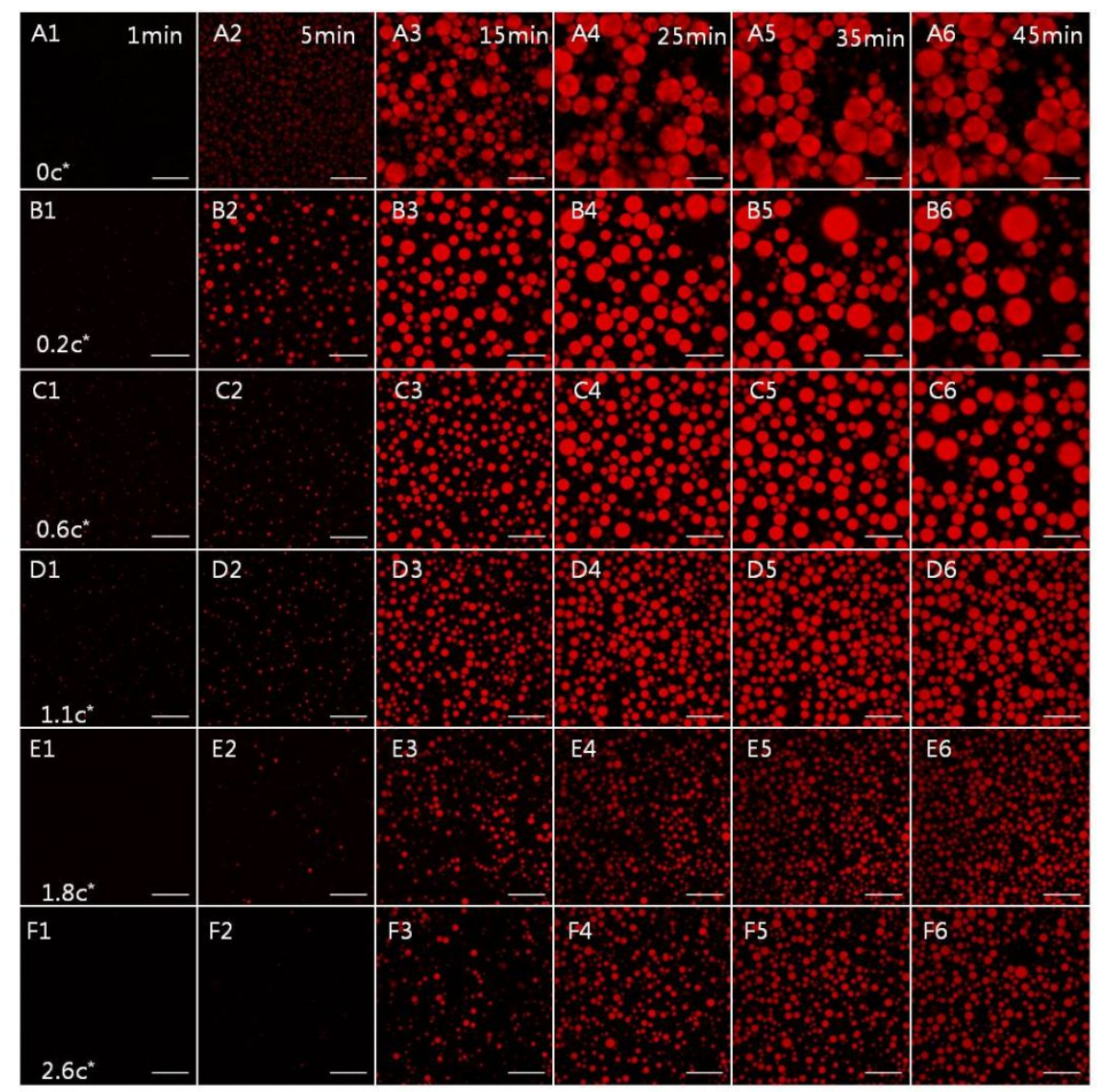

Figure S1. Time dependence of the coacervate formed by $1.0 \mathrm{mg} / \mathrm{mL} \mathrm{S} 5$ and $1.4 \mathrm{mg} / \mathrm{mL}$ ss-oligo in PEO solution at varying concentrations as denoted by $c / c^{*}$. The $c^{*}$ of PEO is $1.7 \mathrm{mg} / \mathrm{ml}$. $1 \times$ DPBS buffer. Scale bar: $25 \mu \mathrm{m}$.
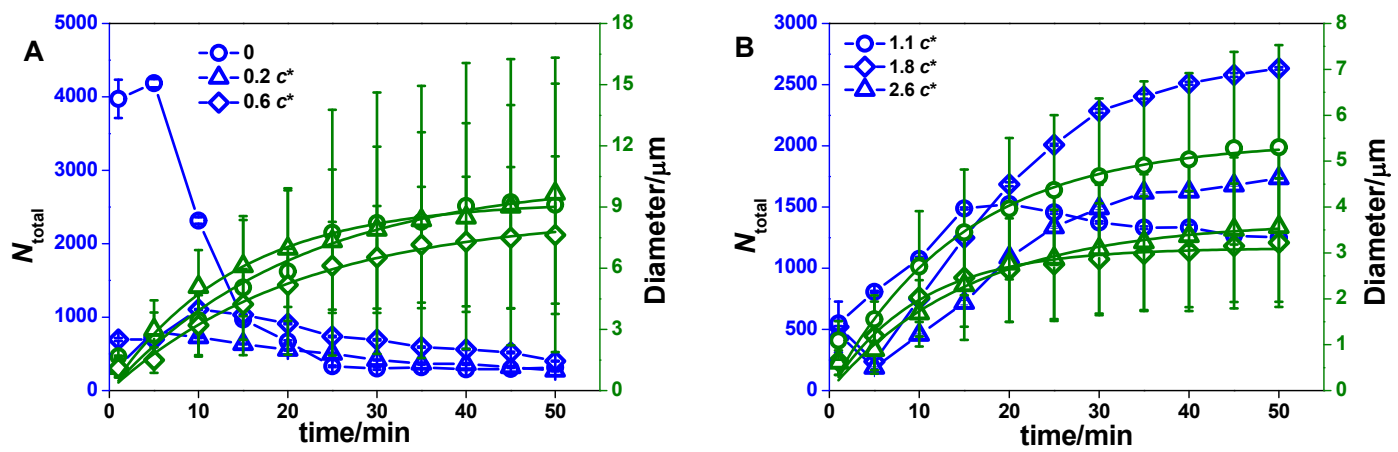

Figure S2. Time dependence of the S5/ss-oligo droplet number and size in (A) DPBS buffer and PEO solution at concentrations lower than $c^{*}$, and (B) PEO solution at concentrations higher than $c^{*}$. The blue lines are used to guide the eyes, while the green lines are the fitting curves by Eq. 3 .

The fluorescence images on S5/ss-oligo coacervation in PEO solutions show a similar pattern as that in PAM solutions. Measurement on the size and number of the droplets 
indicates that the PEO concentration can also be divided into three regimes: $0, c / c^{*}<$ 1 , and $c / c^{*}>1$ (Fig. S1). One major difference is that the increase of the droplet number in semidilute PEO solution, e.g. $1.8 c^{*}$ and $2.6 c^{*}$, is observed in the whole studied time period (Fig. S2), while it is only last about $20 \mathrm{~min}$ in PAM solution at the same concentrations (Fig. 2B), indicating that the coarsening of droplets in semidilute PEO solutions is severely hindered. Given the fact that the $c^{*}$ of PAM $(2.7 \mathrm{mg} / \mathrm{mL})$ is higher than that of PEO $(1.7 \mathrm{mg} / \mathrm{mL})$, the actual concentration of PEO is even lower. The limitation on coarsening indicates that the effect on S5/ss-oligo coacervation by PEO is stronger than that by PAM.

The $m$ values also increase with PEO concentration (Table. S1). However, the range is from 1.3 in PEO solution at $0.2 c^{*}$ to 2.3 in PEO solution at $2.6 c^{*}$, which covers the theoretical value for heterogeneous nucleation (1.5) and close to the theoretical value for homogeneous nucleation (2.5).

The parameter $n$ and $\lg K$ in the growth stage are listed in Table. S2. The $\lg K$ in the growth stage exhibits a similar downward trend as that in the PAM medium, indicating that the diffusion of the droplet-forming unit becomes slower with increasing PEO concentration. The $n$ values also show two distinct values for growth and coarsening, respectively. The average $n$ value for the growth is large than 0.5 in PEO solution at all the studied concentrations, indicating that the excluded volume interaction from PEO is stronger than that from PAM. The $n$ value for coarsening is about 0.3 in PEO solution at $c<1.1 c^{*}$, indicating that BMC is the dominant process. At $1.8 c^{*}$ and $2.6 c^{*}$, the $\mathrm{n}$ values are slightly lower, probably caused by the limited fusion of droplets in PEO network. 
Table S1 $m$ values of the S5/ss-oligo coacervates in PEO medium

\begin{tabular}{ccccccc}
\hline crowder & $\mathbf{0}$ & $\mathbf{0 . 2 c ^ { * }}$ & $\mathbf{0 . 6} \boldsymbol{c}^{*}$ & $\mathbf{1 . 1} \boldsymbol{c}^{*}$ & $\mathbf{1 . 8} \boldsymbol{c}^{*}$ & $\mathbf{2 . 6} \boldsymbol{c}^{*}$ \\
\hline PEO & $1.2 \pm 0.1$ & $1.3 \pm 0.1$ & $1.7 \pm 0.1$ & $1.7 \pm 0.2$ & $1.9 \pm 0.1$ & $2.3 \pm 0.2$ \\
\hline
\end{tabular}

Table S2 Fitting results of the S5/ss-oligo coacervate in PEO medium

\begin{tabular}{ccccccc}
\hline $\boldsymbol{c}$ & $\mathbf{0}$ & $\mathbf{0 . 2} \boldsymbol{c}^{*}$ & $\mathbf{0 . 6} \boldsymbol{c}^{*}$ & $\mathbf{1 . 1} \boldsymbol{c}^{*}$ & $\mathbf{1 . 8} \boldsymbol{c}^{*}$ & $\mathbf{2 . 6} \boldsymbol{c}^{*}$ \\
\hline stage & $0.089 \pm 0.03$ & $0.063 \pm 0.04$ & $-0.47 \pm 0.08$ & $-0.44 \pm 0.06$ & $-0.55 \pm 0.17$ & $-0.73 \pm 0.08$ \\
$\boldsymbol{l g} \boldsymbol{K}$ (growth) & $0.51 \pm 0.06$ & $0.66 \pm 0.03$ & $0.86 \pm 0.05$ & $0.74 \pm 0.04$ & $0.64 \pm 0.07$ & $0.81 \pm 0.05$ \\
$\boldsymbol{n}$ (growth) & $0.32 \pm 0.08$ & $0.37 \pm 0.05$ & $0.34 \pm 0.08$ & $0.30 \pm 0.02$ & $0.26 \pm 0.03$ & $0.26 \pm 0.05$ \\
\hline
\end{tabular}
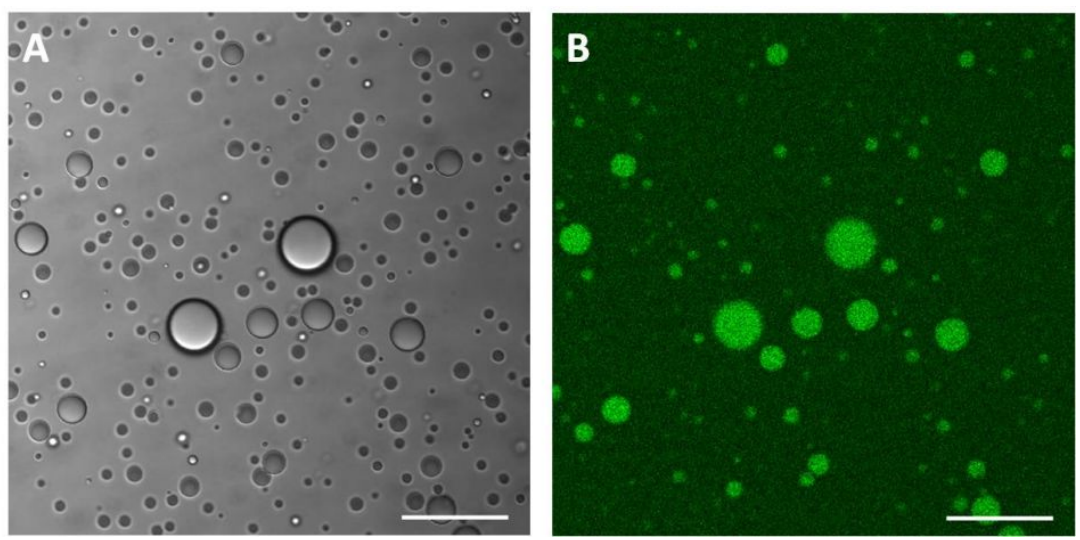

Figure S3. Distribution of FITC-labeled PEG in S5/ss-oligo coacervates. (A) represents the light field and (B) represents the FITC field. Scale bar: $25 \mu \mathrm{m}$.

\section{Supplementary Movies Legends}

Movie S1. Brownian motion coalescence of S5/ss-oligo coacervate droplets in PAM medium at

$0.2 c^{*}$. Movie is shown at $\times 4$ of real-time speed at 2 frames per second. Total duration of recording was $42 \mathrm{~s}$. Scale bar, $5 \mu \mathrm{m}$.

Movie S2. Brownian motion coalescence of S5/ss-oligo coacervate droplets in PAM medium at $0.6 c^{*}$. Movie is shown at $\times 4$ of real-time speed at 2 frames per second. Total duration of recording 
was $32 \mathrm{~s}$ in real time. Scale bar, $5 \mu \mathrm{m}$.

Movie S3. Brownian motion coalescence of S5/ss-oligo coacervate droplets in PAM medium at $1.8 c^{*}$. Movie is shown at $\times 4$ of real-time speed at 2 frames per second. Total duration of recording was 40 s in real time, respectively. Scale bar, $5 \mu \mathrm{m}$. 